\title{
Carcinoma epidermoide oral alrededor de implantes osteointegrados: a propósito de un caso y revisión bibliográfica
}

\author{
Beatriz Carreira Nestares ${ }^{1}$, Marta Alba de Cáceres $^{2,3}$, Alejandro Encinas Bascones ${ }^{4}$, \\ Manuel de Pedro ${ }^{4}$ y Alberto Berguer Sandez ${ }^{4}$
}

Squamous cell carcinoma surrounding osseointegrated dental implants: Clinical case and references review

Introduction: Currently, dental implants is considered as the best choice for edentulism partial or complete treatment. However, this treatment has some associated medical complications such as surrounding gum squamous cell carcinoma. Even though there are not that many cases described in medical literature, it could be appropriated to determine whether there is any relation between this neoplasic disease and the dental implants. Clinical case: Presenting a 85-year-old women with PMH of lichen planus, ex-smoker and osseointegrated dental implant in areas 34, 45 and 46, with surrounding implant gum area Squamous cell carcinoma.

Key words: Implant; Dental; Squamous cell carcinoma.

\section{Resumen}

Introducción: La rehabilitación oral con implantes es en la actualidad la mejor opción para el tratamiento de pacientes parcial o totalmente edéntulos. Sin embargo, no es un procedimiento exento de complicaciones. La aparición de carcinoma epidermoide en la encía circundante de los implantes, aunque infrecuente puede ser una de ellas y aunque no hay muchos casos descritos en la literatura, sería conveniente establecer qué relación, si es que existiera, pueden tener los implantes en el desarrollo de esta enfermedad. Caso clínico: Presentamos el caso de una mujer de 85 años de edad con antecedentes personales de liquen plano oral, exfumadora y portadora de implantes osteointegrados colocados en las áreas correspondientes a 34, 45 y 46, que desarrolló un carcinoma epidermoide en la encía periimplantaria.

Palabras clave: Implantes; Dentales; Carcinoma epidermoide.
${ }^{1}$ Cirugía Bucal e Implantología, Hospital de Madrid, Madrid, España.

${ }^{2}$ Servicio de Anestesiología y Reanimación, Hospital Universitario del Henares, Madrid, España.

${ }^{3}$ Servicio de

Anestesiología, Hospital Universitario Sanitas, La Zarzuela, Madrid, España. 4Servicio de Cirugía Oral y Maxilofacial, Hospital Universitario Clínico San Carlos, Madrid, España.

Recibido el 3 de mayo de 2016, aceptado el 9 de enero de 2017.

Correspondencia a: Beatriz Carreira Nestaresa carreirabeatriz@hotmail.com

\section{Introducción}

La rehabilitación oral con implantes comenzó en la década de los 80 y en la actualidad constituye el tratamiento idóneo de pacientes parcial o totalmente edéntulos. Esta técnica es predecible y tiene altas tasas de éxito, siendo aproximadamente del $90 \%$ a los 5 años ${ }^{1-3}$.

Sin embargo, no es una técnica exenta de complicaciones sobre todo cuando se requieren rehabilitaciones complejas y la aparición de las mismas se ha ido incrementando lógicamente en base al aumento de su uso ${ }^{3}$. Entre las complicaciones más comunes se describe la aparición de inflamación crónica que afecta a los tejidos duros y blandos alrededor de los implantes, esta situación se conoce como periimplintitis ${ }^{3}$. En los últimos años se han observado algunos casos de aparición de carcinoma epidermoide en zonas periimplantarias, y aunque son pocos los casos en la literatura, es conveniente establecer qué relación pueden tener los implantes en el desarrollo de esta enfermedad en pacientes con factores predisponentes, ya que en la mayoría de ellos la enfermedad comienza con los síntomas de la periimplantitis.

La periimplantitis es una dolencia caracterizada por la inflamación y la pérdida de tejido de sostén alrededor de un implante. Esta es una condición ca- 
tabólica con o sin sepsis o supuración. La principal causa de esta enfermedad es el acúmulo de placa dental que se inicia con la gingivitis, más tarde progresa a la formación de bolsas periodontales y finalmente provoca la pérdida de hueso. Otras causas que contribuyen al desarrollo de esta dolencia son: la sobrecarga oclusal y lateral excesiva y/o forzada. Los agentes patógenos implicados son las bacterias anaerobias entre las que destacan principalmente Peptostreptococcus spp. y Fusobacterium spp. ${ }^{2}$. Su tratamiento implica una higiene oral meticulosa, el control de la placa y la administración local de antibióticos.

En la literatura científica, se pone de manifiesto la aparición de carcinoma oral de células escamosas que aparecen en las áreas alrededor de los implantes ${ }^{4}$.

El rol que pueden desempeñar los implantes osteointegrados en el desarrollo del carcinoma epidermoide, si es que lo hubiera, no ha sido bien establecido. En sí mismos, parece que los implantes no presentan potencial carcinogénico, sin embargo, se cree que el estímulo inflamatorio crónico podría representar un papel destacado en el desarrollo de dicha enfermedad. La inflamación crónica ha demostrado ser un factor importante en la carcinogénesis por vía de los mediadores inflamatorios como oxidasas, prostaglandinas, IL-1, IL-6 y TNF-alfa ${ }^{4}$. Dicho estímulo en la cavidad oral podría favorecer la malignización de células de los tejidos periimplantarios. También se baraja la hipótesis de que los implantes podrían ser una vía de penetración de las células tumorales al interior de la medular mandibular al igual que lo sería el ligamento periodontal ${ }^{5}$.

Aquellas periimplantitis que no responden al tratamiento deben ser biopsiadas para descartar un proceso maligno, sobre todo en pacientes que presenten otros factores de riesgo concomitantes ${ }^{1}$.

\section{Caso clínico}

Presentamos el caso de una paciente de 85 años de edad con antecedentes personales de liquen plano, exfumadora desde hace 5 años y portadora de implantes osteointegrados en las áreas correspondientes a 34,45 y 46.

Es remitida al cirujano maxilofacial por parte de su odontólogo al presentarse una lesión ulcerante de $3 \mathrm{~cm}$ de diámetro entre el diente $33 \mathrm{y}$ un implante colocado en el área del diente 34. Dicha lesión es biopsiada y el informe anatomopatológico revela la existencia de un carcinoma epidermoide. El tratamiento consistió en la resección de la lesión con márgenes de seguridad. La anatomía patológica informó márgenes libres del tumor, por lo que se decidió tomar una actitud expectante y mantener el implante. Tras el estudio patológico y de imagen se descarta la presencia de enfermedad locorregional.

En la revisión anual, la paciente presentó molestias en el cuarto cuadrante, por lo que se solicitó una tomografía computarizada que mostró un realce mucoso de $15 \mathrm{~mm}$ en el tercio anterior de la hemilengua derecha con aparente extensión a suelo de boca. No se apreciaron adenopatías sospechosas. Se decidió realizar tratamiento quirúrgico con resección del área afectada y márgenes de seguridad. La anatomía patológica informó carcinoma epidermoide poco diferenciado con bordes de resección libres.

Lamentablemente a los 10 meses la paciente presentó una adenopatía cervical que obligó a realizar vaciamiento cervical funcional derecho y aplicación de radioterapia complementaria. Un mes más tarde aparece en la zona correspondiente a los implantes colocados en área 45 y 46 zona de periimplantitis con área de ulceración y tamaño de $0,4 \mathrm{~cm}$ (Figuras 1 y 2). Se tomó biopsia que confirmó nuevamente la aparición de carcinoma epidermoide. Se decidió nuevamente aplicar tratamiento quirúrgico. Se resecó toda el área mucosa afectada con márgenes de seguridad así como un fragmento óseo que incluyó los implantes (Figura 3). Finalmente, se decidió aplicar quimioterapia para el control de la enfermedad ante la persistencia de recidivas. En las Figuras 4 y 5 se muestran los controles radiológicos postoperatorios.

\section{Material y Método}

Para la realización de la revisión bibliográfica diseñamos un estudio en el que se hizo una revisión sistemática de la evidencia científica disponible en relación con el carcinoma epidermoide en zonas adyacentes a implantes dentales.

Se llevó a cabo una estrategia de búsqueda bibliográfica en las bases de datos y fuentes de información Pubmed/Medline. El rango de búsqueda fue de las referencias publicadas entre enero de 2005 y enero de 2015. La estrategia de búsqueda y palabras clave fueron: «Dental implant AND squamous cell carcinoma». Todas las palabras clave utilizadas para la realización de la búsqueda fueron términos Mesh.

Se seleccionaron los estudios que cumplieron los siguientes criterios:

\section{Criterios de inclusión}

Los criterios de inclusión para la realización de la revisión sistemática fueron: 


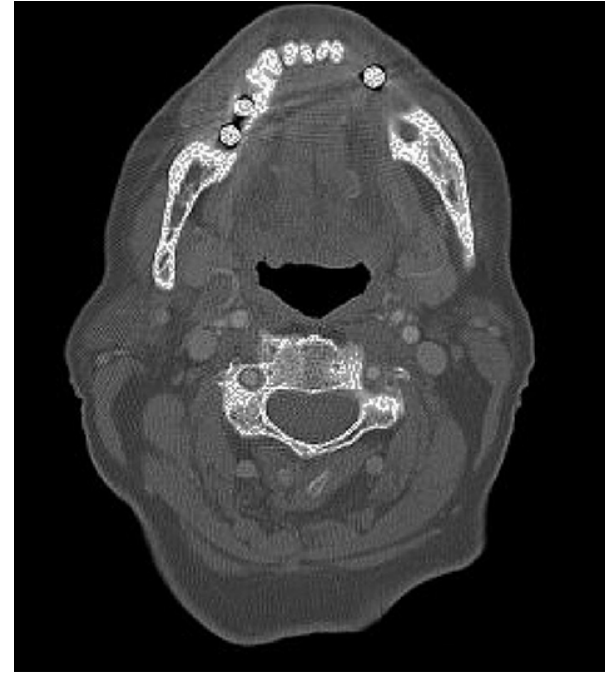

Figura 1. Imagen de tomografía computarizada en la cual se muestra la periimplantitis del cuarto cuadrante.

1. Población de estudio: pacientes con diagnóstico de carcinoma epidermoide en relación con implantes dentales y en su misma localización.

2. Estudios de caso clínico, ensayos clínicos, ensayos clínicos controlados, ensayos clínicos aleatorizados, estudios comparativos y estudios metaanalíticos.

\section{Criterios de exclusión}

Se excluyeron los siguientes estudios:

1. Estudios preclínicos y de laboratorio.

2. Revisiones narrativas, editoriales y artículos de opinión.

3. Presentaciones a congresos (resúmenes y pósteres).

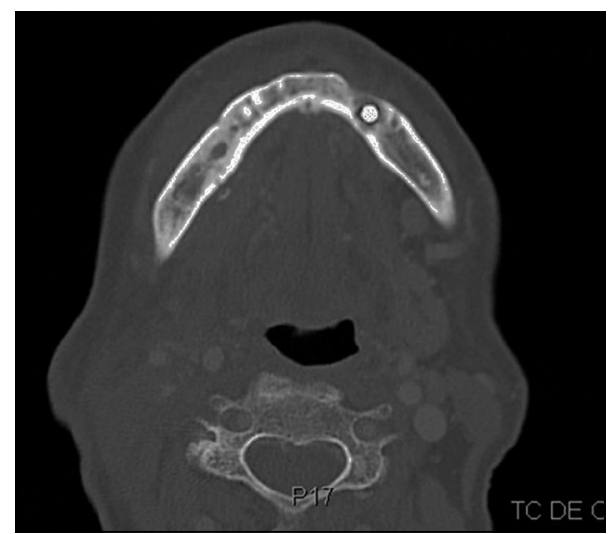

Figura 4. Control con tomografía computarizada posterior a la tercera cirugía.

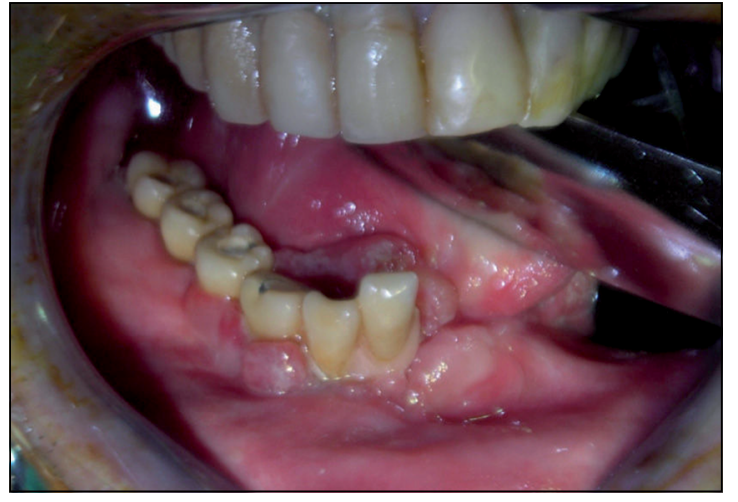

Figura 2. Zona de periimplantitis tumoral en el cuarto cuadrante.

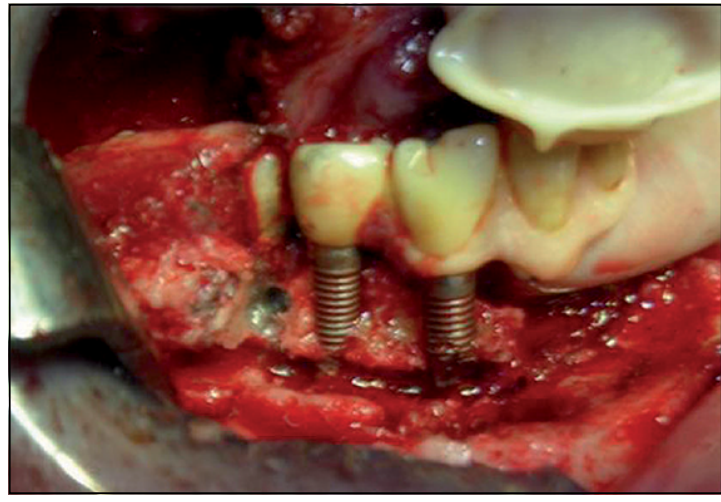

Figura 3. Aspecto del hueso periimplantario en el momento de la resección quirúrgica.

La selección de los estudios fue llevada a cabo por 2 revisores independientes que realizaron una primera lectura de los títulos y resúmenes de los artículos identificados en la búsqueda electrónica. En una reunión de consenso de todos los autores, se realizó la selección final de los artículos que han formado parte de esta revisión.

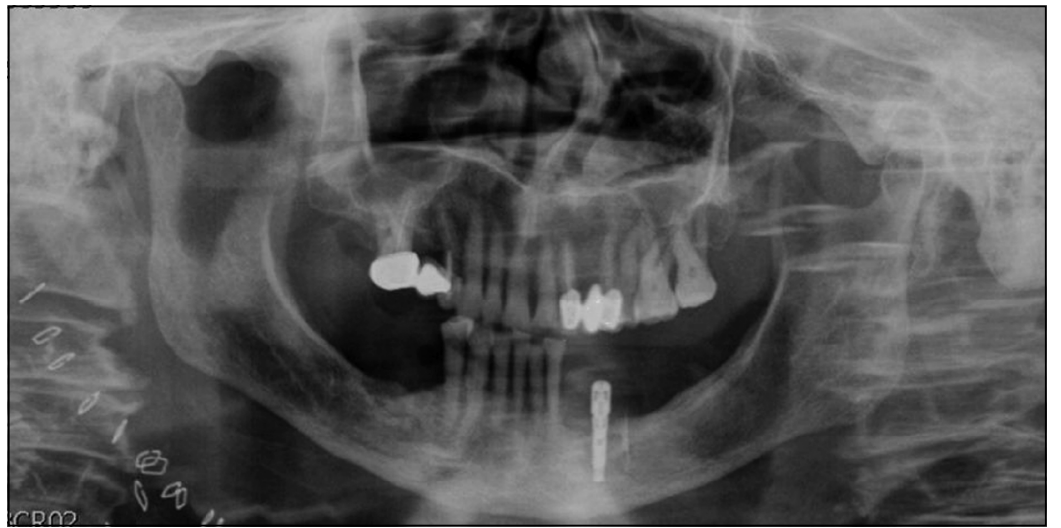

Figura 5. Control OPG después de la extirpación de los implantes del cuarto cuadrante. 


\section{Extracción de datos, variables recogidas y presentación de resultados}

Los datos se extrajeron de manera independiente por 2 revisores con la ayuda de una hoja de recogida de datos diseñada para tal fin. Las variables recogidas para identificar nuestro objetivo fueron: autor, número de pacientes, edad, sexo, localización y hábitos $^{2-13}$ (Tabla 1).

\section{Resultados}

\section{Diagrama de flujo}

La búsqueda electrónica identificó 116 referencias bibliográficas. Tras una primera selección por título y resumen, se identificaron 19 artículos por texto completo, de los que se excluyeron 3 por no cumplir el criterio de inclusión n. ${ }^{\circ}$. Para la recogida de casos se seleccionaron 12 artículos, los cuales registraban en sus casos las siguientes variables: n. ${ }^{\circ}$ de pacientes, sexo, edad, localización, hábitos tóxicos y recurrencia del tumor. Los resultados de la búsqueda se detallan en la Figura 6 .

\section{Síntesis cualitativa}

Fueron encontrados 29 casos de carcinoma epidermoide alrededor de los implantes. En 27 pacientes se localizaba en mandíbula, en 14 de ellos no se especifica la zona, en 6 de los casos estaban localizados en la región anterior mandibular, en 5 de los casos en mandíbula posterior y en uno de los casos tanto en anterior como en posterior. Solo 2 de los casos se localizó en maxilar. Trece casos con carcinoma epidermoide tenían historia anterior de cáncer y 4 de lesiones premalignas como liquen plano oral. La edad media de aparición de la enfermedad fue de 71,7 años. El ratio entre hombres y mujeres fue de 14:15.

Tabla 1. Variables recogidas para identificar nuestro objetivo

\begin{tabular}{|c|c|c|c|c|c|c|}
\hline Autor & $\begin{array}{l}\mathrm{N}^{0} \text { de } \\
\text { casos }\end{array}$ & Edad & Sexo & Localización & Hábitos & $\begin{array}{l}\text { Historia anterior de cáncer } \\
\text { o lesión premaligna }\end{array}$ \\
\hline Gulati et al. $^{2}$ & 1 & 62 & Mujer & Mnd anterior & Fum & Ca Epid \\
\hline Kwok et al. ${ }^{4}$ & 2 & 66,5 & Hombre & $\begin{array}{l}1 \text { Mnd posterior } \\
1 \text { Mnd anterior }\end{array}$ & $\begin{array}{l}1 \text { Fum y alcohol } \\
1 \text { Exfumador pero sí alcohol }\end{array}$ & No \\
\hline Schache et al. ${ }^{5}$ & 1 & 77 & Hombre & Mnd posterior & No dice & No \\
\hline Moergel et al. ${ }^{6}$ & 15 & 66,1 & $\begin{array}{l}8 \text { Mujeres } \\
7 \text { Hombres }\end{array}$ & $\begin{array}{l}14 \text { Mnd } \\
1 \text { Max (zigoma) } \\
4 \text { úlceras } \\
1 \text { Periimplantitis } \\
10 \text { Masas exofíticas }\end{array}$ & 6 Fum y alcohol & $\begin{array}{l}9 \text { Casos antecedentes de } \\
\text { cáncer }\end{array}$ \\
\hline Bhatavadekar et al. ${ }^{8}$ & 1 & 54 & Hombre & Max & No fum & No \\
\hline Meijer et al. ${ }^{9}$ & 1 & 65 & Mujer & Mnd anterior & No referencia & Ca Epid \\
\hline Gallego et al. ${ }^{10}$ & 1 & 70 & Mujer & $\begin{array}{l}\text { Mnd posterior (úlcera, } \\
\text { no periimplantitis) }\end{array}$ & No fum & \\
\hline Gallego et al. ${ }^{11}$ & 1 & 81 & Mujer & Mnd anterior & No fum & $\begin{array}{l}\text { Ca Epid y lesiones } \\
\text { premalignas }\end{array}$ \\
\hline Abu El-Naaj et al. ${ }^{12}$ & 2 & 71 & $\begin{array}{l}1 \text { Hombre } \\
1 \text { Mujer }\end{array}$ & 2 Mnd anterior & $\begin{array}{l}1 \text { No fum } \\
1 \text { Fum }\end{array}$ & 1 LPO 1 Ca Epid \\
\hline Czerninski et al. ${ }^{13}$ & 2 & 66 & $\begin{array}{l}1 \text { Hombre } \\
1 \text { Mujer }\end{array}$ & $\begin{array}{l}1 \text { Mnd posterior } \\
1 \text { Mnd anterior }\end{array}$ & $\begin{array}{l}1 \text { No fum } \\
1 \text { Fum }\end{array}$ & $1 \mathrm{LPO} 1 \mathrm{Ca}$ Epid \\
\hline
\end{tabular}

Ca Epid: carcinoma epidermoide; Fum: fumador; LPO: liquen plano oral; Max: maxilar; Mnd: mandíbula. 


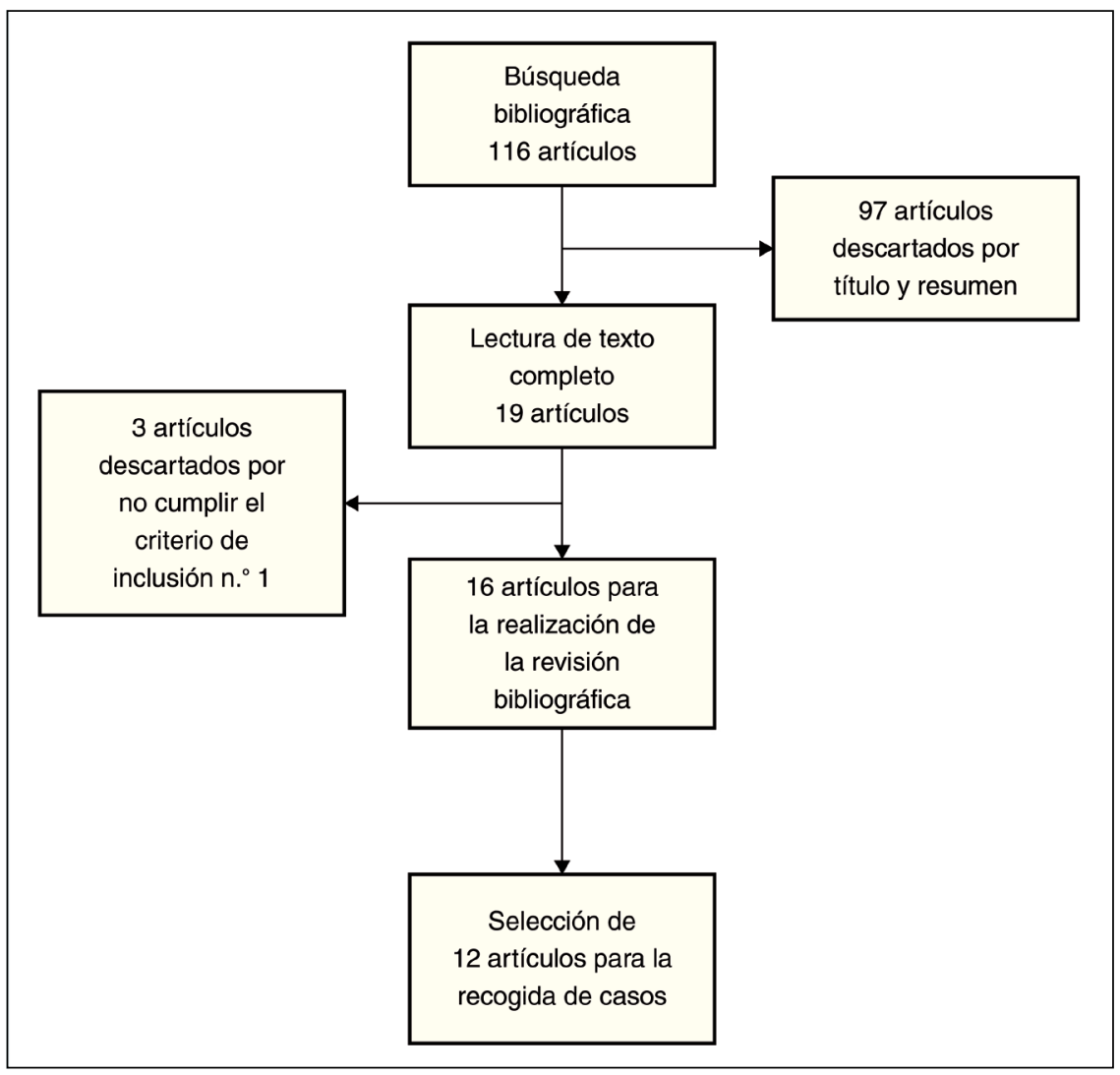

Figura 6. Diagrama de flujo.

\section{Discusión}

Existen pocos casos descritos en la literatura de carcinoma epidermoide diagnosticados en áreas periimplantarias; la baja incidencia no debe causar dificultades para un correcto y rápido diagnóstico que nos lleve a tomar medidas terapéuticas oportunas. Los signos típicos de periimplantitis, con incremento de profundidad de bolsa, pérdida de hueso de soporte y reacción local pueden ser observados en la mayoría de los $\operatorname{casos}^{2-9,11-13}$. Además de los signos clínicos, los hallazgos radiológicos con pérdida vertical de hueso muestran características similares a la pérdida de hueso típica en una periimplantitis convencional. Los únicos signos que pueden hacer sospechar de malignización en un diagnóstico clínico son el tratamiento refractario y el comienzo de síntomas sensitivos como la parestesia.

En el caso presentado por Gallego et al. ${ }^{10}$, la paciente desarrolló un carcinoma epidermoide que podría haber sido provocado por un constante traumatismo debido a la prótesis en extensión (cantiléver) que llevaba sobre los implantes.
Moergel et al. ${ }^{6}$, en su estudio observaron que solo un paciente desarrolló periimplantitis como signo del carcinoma; la mayoría de los casos que estudiaron se diagnosticaron por una masa exofítica alrededor de los implantes y 4 de los pacientes desarrollaron úlceras, como en el caso que hemos presentado.

La etiología del carcinoma epidermoide de cavidad oral es multifactorial e incluye tanto factores relacionados con el estilo de vida (tales como el tabaco, el consumo de alcohol, etc.) como agentes infecciosos tales como el virus del papiloma humano. Los factores dietéticos, como dieta rica en grasas y la dieta baja en frutas y verduras, también se han asociado con un mayor riesgo de cáncer oral ${ }^{13}$.

En el año 1914 T. Hector Boveri propuso la teoría de la mutación somática del cáncer.

Actualmente, se considera que las células cancerígenas son el resultado de una serie de mutaciones que se producen en los genes reguladores. Este hecho ha sido demostrado por Robert A. Weinberg, Geofrey M. Cooper, Michael Whigler y Mamgo Barbaad trabajando en equipos de investigación 
diferentes. Elementos que han demostrado su carcinogénesis como el tabaco actúan sobre los protooncogenes, genes que regulan el crecimiento celular y los transforman en oncogenes.

Aunque el carcinoma epidermoide puede desarrollarse primariamente, a menudo es precedido por lesiones premalignas, tales como la leucoplasia, la eritroplasia o el liquen plano oral. Gulati et al. ${ }^{2}$, Kwok et al. ${ }^{4}$, Moergel et al. ${ }^{6}$, Meijer et al. ${ }^{9}$, Gallego et al. ${ }^{11}$, Abu El-Naaj et al. ${ }^{12}$ y Czerninski et al. ${ }^{13}$ presentaron casos en los que los pacientes tuvieron una historia anterior de carcinoma. Marini et al. ${ }^{7}$, Gallego et al. ${ }^{11}$, Abu El-Naaj et al. ${ }^{12}$, Czerninski et al. ${ }^{13}$ presentaron casos donde los pacientes habían padecido lesiones premalignas, tales como el liquen plano oral. Los autores no establecen un mecanismo de acción concreto, pero todos afirman que la presencia de estas lesiones podría conllevar un riesgo mayor para el desarrollo de la enfermedad. En el caso que presentamos existen antecedentes de liquen plano y de tabaquismo.

El riesgo de un segundo tumor primario en pacientes con tumores del tubo aerodigestivo superior se ha estimado en un 3-7\% anual. A causa de este riesgo, se debe vigilar a estos pacientes de por vida, debiéndose informar a los pacientes de que el consumo continuado de tabaco y alcohol después del tratamiento se ha relacionado con la presencia de segundos tumores primarios del tubo aerodigestivo ${ }^{14,15}$.

Algunos autores hablan de un mayor riesgo para el desarrollo de un tumor en las áreas tratadas con implantes en pacientes con antecedentes de cáncer $^{2,4,9,11-13,16}$. De hecho, de Ceulaer et al. ${ }^{17}$ realizaron un estudio con el propósito de poner en relieve el fenómeno de la recurrencia del tumor alrededor de los implantes. El estudio concluye con que la influencia en los sitios de radiación y los errores en las técnicas quirúrgicas, así como la posibilidad de inducir cambios en la mucosa pueden hacer más sensible de recurrir. Sin embargo, debido al pequeño número de pacientes, no se pueden sacar conclusiones y son necesarios más estudios. Por otro lado, Javed et al. ${ }^{18}$ realizaron una revisión bibliográfica de los casos de carcinoma epidermoide alrededor de los implantes y concluyeron que este tumor es más probable que aparezca en pacientes con una historia previa de cáncer.

El tabaco está reconocido como uno de los principales factores de riesgo para lesiones premalignas orales, así como para el cáncer oral. En nuestra revisión de los 29 casos registrados, solo 12 de los pacientes eran fumadores ${ }^{2,4,6,12,13}$.

Otros autores destacan el consumo de alcohol como un factor de riesgo en el desarrollo de lesio- nes premalignas y del cáncer oral ${ }^{16}$. Kwok et al. ${ }^{4}$, contemplan este factor de riesgo presentando 3 casos donde los individuos que desarrollaron el carcinoma epidermoide eran consumidores de alcohol. Moergel et al. ${ }^{6}$, realizan un estudio retrospectivo y observaron que 6 de los 15 casos presentados eran consumidores de alcohol.

La revisión bibliográfica realizada por Javed et al. ${ }^{18}$ afirma que tanto el tabaco como el alcohol son factores de riesgo para el desarrollo del tumor y no deben pasarse por alto.

Por otro lado, un factor de riesgo que podría explicar el desarrollo de lesiones en pacientes que no tienen hábitos tóxicos podrían ser las deficiencias nutricionales o inmunológicas. Estas podrían explicar la rápida evolución de una lesión. Por ejemplo en casos de gastroplastía se reduce el área de la absorción de algunos nutrientes y vitaminas a nivel del estómago, aumenta la velocidad de tránsito gastrointestinal y también reduce la absorción de ciertos elementos necesarios, los cuales estarían asociados con la menor defensa ante el factor de irritación local de los implantes ${ }^{1}$.

Pfammatter et al. ${ }^{16}$, hicieron un estudio donde analizaban las metástasis y tumores primarios alrededor de los implantes y concluyeron que aquellas periimplantitis que no responden al tratamiento son sospechosas de malignización, por lo que deberían ser biopsiadas según Jané-Salas et al. ${ }^{1}$.

\section{Conclusión}

Según la evidencia publicada revisada no parece que el implante sea de por sí un elemento que cause la formación del carcinoma epidermoide, aunque la inflamación que se produce en los tejidos adyacentes puede ser un factor importante que contribuye al desarrollo de esta enfermedad.

Antes de realizar un tratamiento con implantes, deben analizarse los factores de riesgo del paciente y se debe llevar a cabo una evaluación adecuada del costo-beneficio.

En los pacientes con factores de riesgo, deben realizarse revisiones periódicas. En el caso de encontrar una lesión sospechosa, debe hacerse una biopsia con su consecuente examen histopatológico con el fin de hacer un diagnóstico correcto tan pronto como sea posible.

El carcinoma asociado con los implantes dentales aparece de manera más frecuente en forma de periimplantitis; es por eso que es muy importante realizar un seguimiento riguroso de las zonas periimplantarias con el fin de realizar un 
diagnóstico de carcinoma en su fase inicial si este se produjese.

\section{Responsabilidades éticas}

Protección de personas y animales. Los autores declaran que para esta investigación no se han realizado experimentos en seres humanos ni en animales.

Confidencialidad de los datos. Los autores declaran que han seguido los protocolos de su centro de trabajo sobre la publicación de datos de pacientes.

Derecho a la privacidad y consentimiento informado. Los autores han obtenido el consentimien- to informado de los pacientes y/o sujetos referidos en el artículo. Este documento obra en poder del autor de correspondencia.

\section{Financiación}

El presente trabajo no posee fuente de financiación.

\section{Conflicto de intereses}

Los autores declaran no tener ningún conflicto de intereses.

\section{Bibliografía}

1. Jané-Salas E, López-López J, RosellóLlabrés X, Rodríguez- Argueta OF, Chimenos-Küstner E. Relationship between oral cancer and implants: Clinical cases and systematic literature review. Med Oral Patol Oral Cir Bucal. 2012;17:23-8.

2. Gulati A, Puthussery FJ, Downie IP, Flood TR. Squamous cell carcinoma presenting as peri-implantitis: A case report. Ann R Coll Surg Engl. 2009;91:8-10.

3. Eguía del Valle A, Martínez-Conde Llamosas R, López Vicente J, Uribarri Etxebarria A, Aguirre Urizar JM. Primary oral squamous cell carcinoma arising around dental osseointegrated implants mimicking peri-implantitis. Med Oral Patol Oral Cir Bucal. 2008;13:489-91.

4. Kwok J, Eyeson J, Thompson I, McGurk M. Dental implants and squamous cell carcinoma in the at risk patient-report of three cases. Br Dent J. 2008;205:543-5.

5. Schache A, Thavaraj S, Kalavrezos N. Osseointegrated implants: A potential route of entry for squamous cell carcinoma of the mandible. Br J Oral Maxillofac Surg. 2008;46:397-9.

6. Moergel M, Karbach J, Kunkel M, Wagner W. Oral squamous cell carcinoma in the vicinity of dental implants. Clin Oral Investig. 2014;18:277-84.

7. Marini E, Spink MJ, Messina AM. Peri-implant primary squamous cell carcinoma: A case report with 5 years' follow-up. J Oral Maxillofac Surg. 2013;71:322-6.

8. Bhatavadekar NB. Squamous cell carcinoma in association with dental implants: An assessment of previously hypothesized carcinogenic mechanisms and a case report. J Oral Implantol. 2012;38:792-8.

9. Meijer GJ, Dieleman FJ, Bergé SJ, Merkx MA. Removal of an oral squamous cell carcinoma including parts of osseointegrated implants in the marginal mandibulectomy. A case report. Oral Maxillofac Surg. 2010;14:253-6.

10. Gallego L, Junquera L, Llorente S. Oral carcinoma associated with implantsupported overdenture trauma: A case report. Dent Traumatol. 2009;25:e3-4.

11. Gallego L, Junquera L, Baladrón J, Villarreal P. Oral squamous cell carcinoma associated with symphyseal dental implants: An unusual case report. J Am Dent Assoc. 2008;139:1061-5.

12. Abu El-Naaj I, Trost O, Tagger-Green N, Trouilloud P, Robe N, Malka G, et al. Peri-implantitis or squamous cell carcinoma? Rev Stomatol Chir Maxillofac. 2007;108:458-60.

13. Czerninski R, Kaplan I, Almoznino G, Maly A, Regev E. Oral squamous cell carcinoma around dental implants. Quintessence Int. 2006;73:707-711.

14. Khuri FR, Lippman SM, Spitz MR, Lotan R, Hong WK. Molecular epidemiology and retinoid chemoprevention of head and neck cancer. J Natl Cancer Inst. 1997;89:199-211.

15. León X, Quer M, Diez S, Orús C, LópezPousa A, Burgués J. Second neoplasm in patients with head and neck cancer. Head Neck. 1999;21:204-10.

16. Pfammatter $\mathrm{C}$, Lindenmüller IH, Lugli A, Filippi A, Kühl S. Metastases and primary tumors around dental implants: A literature review and case report of peri-implant pulmonary metastasis. Quintessence Int. 2012;43:563-70.

17. De Ceulaer J, Magremanne M, van Veen A, Scheerlinck J. Squamous cell carcinoma recurrence around dental implants. J Oral Maxillofac Surg. 2010;68:2507-12.

18. Javed F, Al-Askar M, Qayyum F, Wang HL, Al-Hezaimi K. Oral squamous cell carcinoma arising around osseointegrated dental implants. Implant Dent. 2012;21:280-6. 